\title{
Complex brain circuits studied via simultaneous and permanent detection of three transported neuroanatomical tracers in the same histological section
}

\author{
J.L. Lanciego ${ }^{\text {a,* }}$, F.G. Wouterlood ${ }^{\text {b,c }}$, E. Erro ${ }^{\text {a }}$, J. Arribas ${ }^{\text {a }}$, N. Gonzalo ${ }^{\text {a }}$, X. Urra ${ }^{\text {a }}$, \\ S. Cervantes ${ }^{\text {a }}$, J.M. Giménez-Amaya ${ }^{\text {a }}$ \\ a Departamento de Anatomía, Facultad de Medicina, Universidad de Navarra, Irunlarrea No 1, 31080 Pamplona, Spain \\ ${ }^{\mathrm{b}}$ Graduate School Neurosciences Amsterdam, Research Institute Neurosciences, Vrije Universiteit, Amsterdam, The Netherlands \\ ${ }^{\mathrm{c}}$ Department of Anatomy and Embryology, Faculty of Medicine, Vrije Universiteit, 7 v.d. Boechorststraat, \\ 1081 BT Amsterdam, The Netherlands
}

Received 15 March 2000; accepted 9 July 2000

\begin{abstract}
Experimental neuroanatomical tracing methods lie at the basis of the study of the nervous system. When the scientific question is relatively straightforward, it may be sufficient to derive satisfactory answers from experiments in which a single neuroanatomical tracing method is applied. In various scientific paradigms however, for instance when the degree of convergence of two different projections on a particular cortical area or subcortical nucleus is the subject of study, the application of single tracing methods can be either insufficient or uneconomical to solve the questions asked. In cases where chains of projections are the subjects of study, the simultaneous application of two tracing methods or even more may be compulsory. The present contribution focuses on combinations of several neuroanatomical tract-tracing strategies, enabling in the end the simultaneous, unambiguous and permanent detection of three transported markers according to a three-color paradigm. A number of combinations of three tracers or of two tracers plus the immunocytochemical detection of a neuroactive substance can be conceived; we describe several of these combinations implemented by us using the present multitracer protocol. (c) 2000 Elsevier Science B.V. All rights reserved.
\end{abstract}

Keywords: Chromogen; Immunocytochemistry; Dextran amine; Cholera toxin; Fluoro-Gold; Axonal tracing; Neuroanatomy

\section{Introduction}

Neuroanatomical tract-tracing methods are powerful tools for the study of neuronal circuits. Tract-tracing allows us to study in detail the origins, courses and patterns of termination of the fiber projections interconnecting brain areas. The history of experimental tracing by transport is rather short, beginning in the 1970s with the introduction of horseradish peroxidase as the first of the retrogradely transported substances (Kristensson et al., 1971; Mesulam, 1976, 1978); the autoradiographic tracing method in conjunction with uptake of radioactive aminoacids, incorporation into

* Corresponding author. Tel.: + 34-48-425600, ext. 6396; fax: + 34-48-425649.

E-mail address: jlanciego@unav.es (J.L. Lanciego). proteins and subsequent transport (Hendrickson, 1969) will not be further considered in this review. During the past three decades, many substances have been successfully introduced as neuroanatomical tracers and the current technical arsenal contains a wide variety of transported markers (for review, see textbooks by Cowan and Cuénod, 1975; Heimer and Robards, 1981; Heimer and Záborsky, 1991; Bolam, 1992). Several of these markers can be used for the verification of the existence of synaptic connectivity by electron microscopy (see van Haeften and Wouterlood, this issue).

\subsection{Complex circuits: the need for combined methods}

As a part of our ongoing efforts trying to elucidate the relationships underlying basal ganglia circuits in 
normal and pathologically affected brains such as in Parkinson's disease and other neurodegenerative disorders, strategies are necessary aimed at the simultaneous visualization of several of these complex circuits in the same tissue section. Illustrative examples of complex circuits may also be found elsewhere the central nervous system. Our interest in the basal ganglia was to elucidate whether a specific fiber input to a particular area segregates or converges on one or two separate populations of identified projection neurons and, if convergence occurs, to what degree patterns of overlap of the projections exist.

Amenable procedures attempting to disclose three transported markers have to meet several demands. First, the involved tracers should belong to the same general family (fluorescent vs non-fluorescent tracers). Second, the speed with which uptake and transport occurs should be in the same range, and the survival times be in such a range that there should be no need for multiple surgical sessions. Third, the tracers should preferably be either obligatory anterograde or retrograde. Fourth, commercially available antisera for the immunodetection of each individual tracer should not cross-react with each other or in any other way interfere with the detection of the other involved markers. Fifth, individual chromogens should be available that differ in color or aspect in such a way that unequivocal discrimination is possible. Sixth, the colored precipitates produced during the combined staining procedure should preferably be permanent. Finally, follow-up by taking sections to the electron microscopy should be a possible option. Taken these demands into consideration one can predict that, despite the large number of neuroanatomical probes currently available in the existing technical arsenal, only a few possible candidate tracing/detection/chromogen methods may be available for the purpose of combination in a multiple tracing protocol (Fig. 1). Therefore the search was and is still going on to find even better and more compatible neuroanatomical tracers, detection methods and chromogens than those already at our disposal. Among the currently available transported markers we have selected particularly versatile and stable tracers, notably most promising, the anterogradely transported tracer biotinylated dextran amine (BDA; Veenman et al., 1992; Brandt and Apkarian, 1992; Reiner et al., 1993; Rajakumar et al., 1993; Lanciego and Wouterlood, 1994), the retrogradely transported tracer Fluoro-Gold (FG; Schmued and Fallon, 1986; Schmued and Heimer, 1990; Wessendorf, 1991; Schmued, 1994) and the bi-directionally transported tracer cholera toxin, $\beta$ subunit (CTB; Stoeckel et al., 1977; Trojanowski et al., 1981, 1982; Wan et al., 1982; Ericson and Blomqvist, 1988).

\subsection{Anterogradely transported tracer: biotinylated dextran amine $(B D A)$}

In retrospect, the introduction of the family of dextran amines as neuroanatomical tracing substances in the mid-1980s (Glover et al., 1986), was a great step forward in neurobiology. The introduction as a tracer by Veenman and co-workers (1992) of the biotinylated derivative (BDA, biotinylated dextran amine) can be considered a breakthrough. BDA proved to satisfy nearly all the major criteria of an ideal tracer with characteristics that appeared very flexible in combination with another tracers in multiple protocols: easy application, quick onset of transport and broad range of transport time, permanent visualization with no background and, very important, no need for the use of antisera throughout the staining procedure, since its visualization is based on biotin-avidin interactions. The additional benefit of BDA is that, when applied for electron microscopic analysis, transported BDA is far less difficult to visualize than other commonly used anterograde tracers, for instance the lectin Phaseolus vulgaris leucoagglutinin (PHA-L) (Wouterlood and Jorritsma-Byham, 1994).

\subsection{Retrogradely transported tracer: $F G$}

The retrogradely transported marker FG was introduced in the same year as the dextran amines (Schmued and Fallon, 1986). Because of its excellent and stable fluorescence, simplicity of application, easy reproducibility of the results and many other unique properties, this fluorescent dye has become widely used for tracing neuronal connections (Pieribone and Aston-Jones, 1988; Schmued and Heimer, 1990; Divac and Mogensen, 1990; Wessendorf, 1991; Akintunde and Buxton, 1992; Schmued, 1994; Novikova et al., 1997). Drawbacks with respect to the demands for multiple tracing listed above is that this dye belongs to the family of fluorescent tracers, and that FG is not electrondense in itself and therefore is not very useful in ultrastructural analysis without the help of labor- and time-consuming post-staining processing like photoconversion (Bentivoglio and Su, 1990; Balercia et al., 1992). In this regard, the introduction of sensitive antisera against FG has allowed 'change of family' and opened the possibility of using a permanent chromogen in the light microscopical detection of the dye (Chang et al., 1990; Van Bockstaele et al., 1994). The consequence of this development meant the opening of a wider spectrum of possibilities with this retrograde tracer when used in combined methodologies (Lanciego et al., 1997, 1998a,b; Lanciego and Giménez-Amaya, 1999) as well as in electron microscopic applications. 


\subsection{Bi-directionally transported tracer: $C T B$}

The bi-directionally transported tracer cholera toxin, B subunit (CTB; Stoeckel et al., 1977) has proven to be a reliable marker in tracing strategies both in peripheral nervous structures and in the central nervous system. Its detection can be combined with other immunocytochemical procedures, for instance that for neurotransmitter identification (Luppi et al., 1990) or neuropeptide detection (Luppi et al., 1987). Combination of CTB tracing is also possible with PHA-L tracing (Bruce and Grofova, 1992; Vetter et al., 1993). CTB has occasionally been used for anterograde tracing purposes. The quality of fiber labeling with CTB is poor however, compared to other standard anterograde fiber tracers, although several procedures have been devised in attempts to solve this drawback (Angelucci et al., 1996).

Once the neuroanatomical tracers to be used in a combined multi-tracer experimental procedure had been selected, the next step was to find chromogens suitable for the unequivocal discrimination of each individual tracer substance. It seems perhaps trivial, but it should be stressed here that a major demand in triple staining is that each chromogen does not interact with the other chromogens and with any of the chemicals used in detecting the other two tracers. There is a large body of literature dealing with the combination of at least two distinct chromogens for light and electron microscopy (Hsu and Soban, 1982; Liposits et al., 1983; Van den Pol, 1985; Görcs et al., 1986; Lakos and Basbaum, 1986; Levey et al., 1986; Norgren and Lehman, 1989; Smith and Bolam, 1992; Dolleman-van der Weel et al., 1994; Zhou and Grofova, 1995). Although a large number of peroxidase substrates is available (Trojanowski et al., 1983), only few of these substrates fulfil vital requirements in triple labeling methods such as good color discrimination between the precipitates, handling safety, compatibility through dehydration procedures and the specificity of their respective textures in the electron microscopy. Chromogens such as diaminobenzidine (DAB) and V-VIP (trade mark of Vector Laboratories) may be compatible with such criteria. At the light microscopic level, the combined use of nickel-enhanced DAB (DAB-Ni, blue-black precipitate; introduced by Hancock, 1982), non-intensified DAB (brown reaction product) and V-VIP (purple precipitate) has proven to produce reliable, well discernible and permanent colors (cf. Lanciego et al., 1997, 1998a,b).

Having selected tracers and chromogens, a multitracer protocol could be developed and tested. In this contribution we discuss two examples of multiple tracing combinations: (i) a triple-tracing protocol including anterograde BDA tracing with retrograde FG tracing and bi-directional CTB tracing; (ii) a triple protocol in which anterograde BDA tracing is combined with ret- rograde FG tracing (or with bi-directional CTB tracing) and with enzyme immunocytochemistry for the detection of tentative target neurons with a particular chemical identity (by parvalbumin- or tyrosine hydroxylase-immunocytochemistry) of the anterogradely labeled projections.

The detection of BDA depends on the irreversible reaction of biotin with chromogen-tagged avidin. It can be carried out largely independent of immunocytochemical procedures. Because of this characteristic, other combinations of two tracing methods and tracer-, transmitter-, neuropeptide-, or enzyme immunocytochemistry, and a biotin-based detection can easily be conceived. Researchers may be interested, for example, in the chemical identity of postsynaptic target neurons of particular projection fibers rather than in identifying three distinct circuits. In another experiment one might want to visualize one afferent system, one efferent system and the chemical nature of, for example, neurons containing the retrogradely transported marker, interneurons located in close vicinity to those displaying the transported tracers, or use a general immunocytochemical marker to delineate the brain area in which the labeled neurons and/or fibers are located. To underscore those aims we illustrate our three-color paradigm with two combinations. First we deal with the combination of anterograde BDA tracing, bi-directional CTB tracing and the immunocytochemical staining of neurons containing tyrosine hydroxylase. Then we discuss a similar combined procedure to stain anterogradely transported BDA, retrogradely transported FG and immunocytochemistry for the detection of parvalbumin-containing interneurons.

\subsection{Added value of triple staining procedures}

Neuroanatomical studies in primates are very demanding, time consuming and particularly expensive. For instance, three isolated animal experiments, each conducted with a single tract-tracing strategy, will produce valuable data on the organization of each single projection. In order to establish the degree of convergence/divergence existing among the involved circuits it will however be necessary to conduct supplementary experiments. Furthermore, animal testing (especially when conducted in primates) is under increasing critical observation by the society at large. Overall, application of triple staining procedures may produce more information in one experimental animal about the organization of the involved brain circuits than three separate experiments in three experimental subjects. Thus a marked reduction may be obtained in the number of animal used and additionally more information gained about the organization of the brain circuits under study. 


\section{Materials and methods}

The three-color paradigm described here should be of considerable use to neuroanatomists. There are also a number of procedural findings that will be very attractive to investigators who use any multiple tracing/staining strategy.

In order to ensure replication of the method, several 'technical tips' for histological tissue processing are given below. The reported method is dealing with the simultaneous visualization of anterogradely transported BDA in combination with retrogradely transported FG and bi-directionally transported CTB. Minor changes (mainly related to appropriate antisera selection) have to be made in case of selecting different tracers or a combination of tracers with the immunocytochemical detection of neuroactive substances. More extensive information dealing with other satellite issues (tracer delivery, survival time, perfusion and fixation) can be obtained elsewhere (cf. Lanciego et al., 1997, 1998a,b).

\subsection{Tracer delivery}

BDA (Molecular Probes, Eugene, OR) and FG (Fluorochrome, Englewood, CA) were delivered either by iontophoresis (rats) or by pressure (in primates, via a Hamilton syringe). BDA was iontophoretically injected as a $10 \%$ solution in $10 \mathrm{mM} \mathrm{PB}, \mathrm{pH} 7.25$, through a glass micropipette (inner tip diameter $20-35 \mu \mathrm{m}$ ) using a positive-pulsed direct current ( $7 \mathrm{~s}$ on/off) for $10 \mathrm{~min}$. Next, a $2 \%$ solution of $\mathrm{FG}$ in $100 \mathrm{mM}$ cacodylate buffer, $\mathrm{pH} 7.3$ was delivered in the appropriate target area using the same parameters described above for the BDA injections. Finally, $1-5 \mu$ of CTB (List Biological Laboratories, Campbell, CA) was pressure-injected in the putamen as a $2 \%$ solution in $0.1 \mathrm{M} \mathrm{PB}, \mathrm{pH}$ 6.0. BDA and FG were pressure-injected only when dealing with primates. In such cases, each primate has received one single injection in the medial part of the globus pallidus of $1 \mu \mathrm{l}$ of BDA $(10 \%$ solution in $10 \mathrm{mM} \mathrm{PB}$, $\mathrm{pH}$ 7.25) and three injections in the caudate nucleus of $2 \mu \mathrm{l}$ of $\mathrm{FG}(2 \%$ solution in $100 \mathrm{mM}$ cacodylate buffer, $\mathrm{pH}$ 7.3).

\subsection{Survival time}

The survival time has to be adapted to the length of the circuits being studied. All the tracers used in the present experimental paradigm have a range of survival times sufficiently broad to facilitate their appropriate combination in a triple-labeling method with no need for multiple surgery. In our hands, when considering rats as experimental subjects, one week is a survival time long enough to provide a nice staining quality of the injected tracers, although tract-tracing with BDA, CTB and FG still display good labeling quality when survival times of up to 4 weeks are being used. In primates, good results were obtained using standard survival of two weeks. Longer survival times were never tested by us; nice results were reported by Brandt and Apkarian (1992) with BDA tract-tracing in primates using a survival time of up to seven weeks. FG can be used at a wider range of survival time, ranging from 2 days to 1 year (Schmued and Fallon, 1986; Pieribone and Aston-Jones, 1988; Schmued and Heimer, 1990; Akintunde and Buxton, 1992; Schmued, 1994; Novikova et al., 1997). Regarding CTB, one should keep in mind that a short survival time will increase the amount of anterograde CTB labeling (Trojanowski et al., 1981; Angelucci et al., 1996), while retrograde labeling with CTB seems to be enhanced with longer survival times.

\subsection{Perfusion, fixation, cryoprotection and sectioning}

The animals were anesthetized with an overdose of $10 \%$ chloral hydrate in distilled water and perfused transcardially. First, a brief vascular rinse was conducted with a Ringer saline solution followed by 1000 $\mathrm{ml}$ (rats) or $3000 \mathrm{ml}$ (primates) of cold fixative containing $4 \%$ freshly depolymerized paraformaldehyde, $0.1 \%$ glutaraldehyde and $0.2 \%$ of saturated picric acid in 125 $\mathrm{mM} \mathrm{PB}, \mathrm{pH}$ 7.4. In case of primates, perfusion was continued with $1000 \mathrm{ml}$ of a cryoprotective solution (prepared according to Rosene et al., 1986) containing $10 \%$ glycerin and $1 \%$ DMSO in $125 \mathrm{mM} \mathrm{PB}, \mathrm{pH} 7.4$. After the perfusion, the brains were stored $48 \mathrm{~h}$ in a cryoprotective solution containing $20 \%$ glycerin and $2 \%$ DMSO in PB 125 mM, pH 7.4. Finally, tissue sections (40 $\mu \mathrm{m}$ thick) were obtained on a sliding microtome (Microm) and stored in the second cryoprotective solution (deep freezer, $-80^{\circ} \mathrm{C}$ ) awaiting further histological processing.

\subsection{Histology}

The BDA protocol was carried out first, according to the procedure of Veenman et al. (1992). Sections were incubated in the $\mathrm{ABC}$ solution (Vector Labs; $8 \mu \mathrm{l}$ of solution $\mathrm{A}$ in $1 \mathrm{ml}$ of $0.05 \mathrm{M}$ TBS-Tx pH 8.0 and mix well; then add $8 \mu \mathrm{l}$ of solution B, mix well and wait 30 min prior to use) for $90 \mathrm{~min}$ at room temperature (RT). Next, sections were reacted for 5-10 min in the nickelenhanced $\mathrm{DAB} / \mathrm{H}_{2} \mathrm{O}_{2}$ solution (DAB-Ni; DAB from Sigma, nickel ammonium sulphate from Farmitalia Carlo Erba). DAB-Ni solution was prepared by dissolving $200 \mathrm{mg}$ nickel ammonium sulphate in $50 \mathrm{ml}$ of 0.05 $\mathrm{M}$ Tris/HCl, $\mathrm{pH}$ 8.0. Next, add $0.0075 \mathrm{~g}$ of DAB. Then, $10 \mu \mathrm{l}$ of $30 \% \mathrm{H}_{2} \mathrm{O}_{2}$ was added just prior to use. Once the BDA protocol was completed, the next step consisted of the incubation $\left(60 \mathrm{~h}, 4^{\circ} \mathrm{C}\right)$ in a cocktail of primary antisera comprising 1:2000 goat anti-CTB (List 


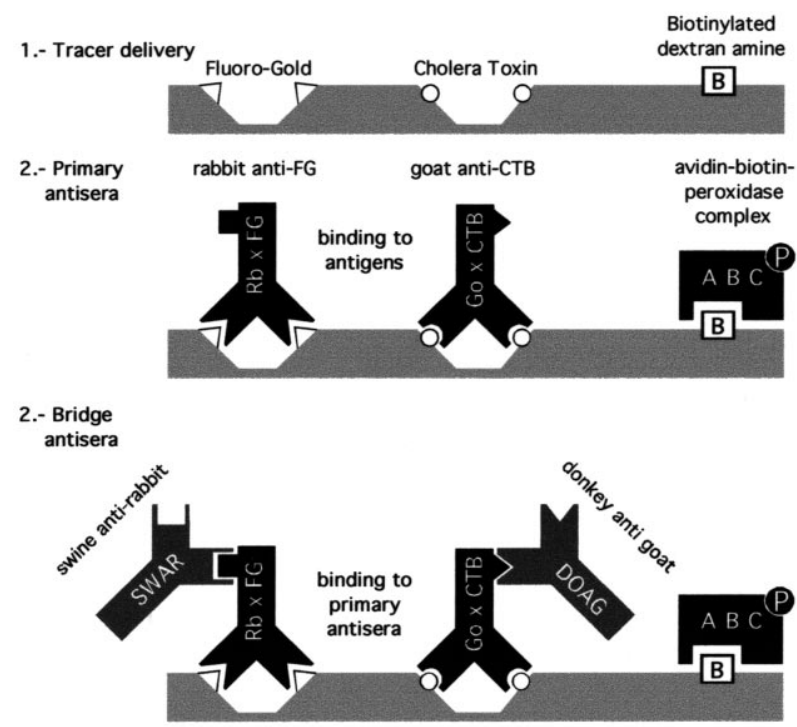

3.- PAP complexes

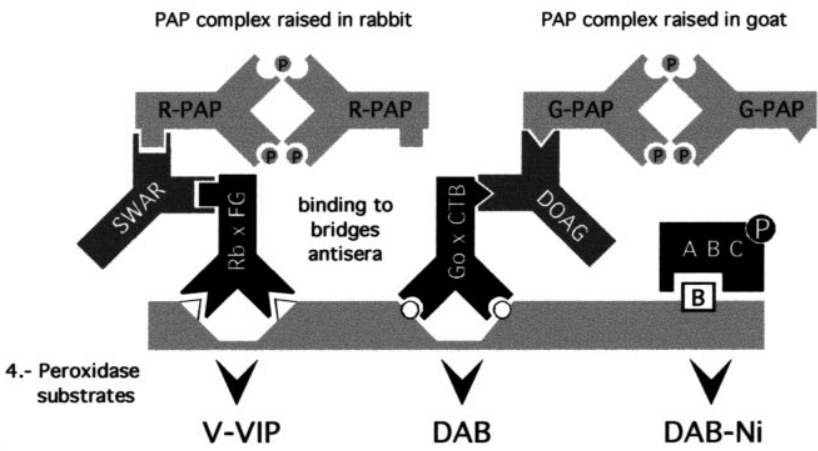

Fig. 1. Flow chart illustrating the different antisera and complexes used in the present study and their respective binding sites (modified after Smith and Bolam, 1992). First, transported BDA is histochemically visualized by means of an $\mathrm{ABC}$ solution and then stained black (DAB-Ni as chromogen). Subsequently, two PAP procedures are conducted simultaneously for the detection of transported CTB and FG. CTB is stained brown with DAB and FG is stained purple using the peroxidase substrate V-VIP. When trying to achieve a good final technical quality, it is extremely important in what order the different chromogens are applied. The best quality is obtained using first DAB-Ni substrate, then the regular DAB solution and finally the peroxidase substrate V-VIP.

Biological Labs) and 1:2000 rabbit anti-FG (Chemicon). Next, sections were incubated $(2 \mathrm{~h}, \mathrm{RT})$ in a cocktail solution of bridge antisera containing 1:50 donkey anti-goat (Sigma) mixed with 1:50 swine antirabbit (Dako). The next step consisted of the incubation in a peroxidase anti-peroxidase complex raised in goat (1:600 G-PAP, Sigma; $90 \mathrm{~min}, \mathrm{RT})$. The transported CTB was then visualized using DAB as the chromogen $(10 \mathrm{mg}$ of DAB in $10 \mathrm{ml}$ of $0.05 \mathrm{M}$ Tris/ $\mathrm{HCl}, \mathrm{pH} 7.6$; once dissolved and filtered, add $3.3 \mu \mathrm{l}$ of $\mathrm{H}_{2} \mathrm{O}_{2}$ and incubate for $20-30$ min under microscopical monitoring). Transported FG was finally visualized by incubating the sections in a PAP complex prepared in rabbit (1:600 R-PAP, Dako; $90 \mathrm{~min}, \mathrm{RT}$ ), followed by immunoreaction with V-VIP solution (1 drop of each vial in $3.5 \mathrm{ml}$ of $0.05 \mathrm{M}$ Tris $/ \mathrm{HCl}, \mathrm{pH} 7.6$, and incubate for 5-10 min). The final resulting V-VIP solution is diluted twice as compared to the original recipe as provided by the supplier. Please note that a more diluted peroxidase substrate results in a much better manageable chromogen. This makes it easier to monitor the progress of the reaction to avoid typical drawbacks such as background staining and color mixing phenomena. Once the entire procedure was finished, the stained sections were transferred to a freshly prepared, lukewarm solution of $2 \%$ gelatin in $0.05 \mathrm{M}$ Tris $/ \mathrm{HCl}$, $\mathrm{pH}$ 7.6, mounted on glass slides, dried thoroughly, quickly dehydrated in toluene and coverslipped with Entellan (Merck).

All antisera were always prepared in a buffer consisting of $0.05 \mathrm{M}$ TBS-Tx, pH 8.0. Primary antisera were implemented with a $2 \%$ solution of bovine serum albumin (BSA, Merck). Throughout the procedure, extensive washing between steps with $0.05 \mathrm{M}$ TBS-Tx was conducted. Several rinsing steps with $0.05 \mathrm{M}$ Tris $-\mathrm{HCl}$, $\mathrm{pH} 7.6$ were carried out prior to and after the incubations in the different chromogen solutions.

\section{Results}

In primates, our surgical procedure was aimed at delivery of BDA in the medial part of the globus pallidus, while CTB and FG were injected in the caudate nucleus and in the putamen, respectively. As a result of the present procedure, thick BDA-labeled fibers were found in the substantia nigra, pars reticulata. A smaller population of thin BDA-labeled fibers was seen extending into the substantia nigra, pars compacta $(\mathrm{SNc})$. Within $\mathrm{SNc}$, two different subpopulations of CTB- or FG-labeled neurons were disclosed (brownvs purple-stained neurons, respectively). Both kinds of labeled neurons displayed a marked tendency to be distributed in separate clusters (Fig. 2A). Terminal fields of thin BDA-labeled fibers were seen to converge within thalamic nuclei (mainly the centromedianparafascicular complex) containing clusters of CTB-labeled neurons. In order to obtain an accurate localization of CTB-labeled neurons within SNc, a combination of anterograde BDA tracing, retrograde CTB tracing and immunocytochemistry using antisera against tyrosine hydroxylase was designed, as illustrated in Fig. 3A.

Our ongoing research efforts also comprise the study of the target structures of efferent projections from the perirhinal cortex to the entorhinal cortex in rats. We are interested in elucidating whether perirhinal efferent axons contact principal cells in the superficial layers of 

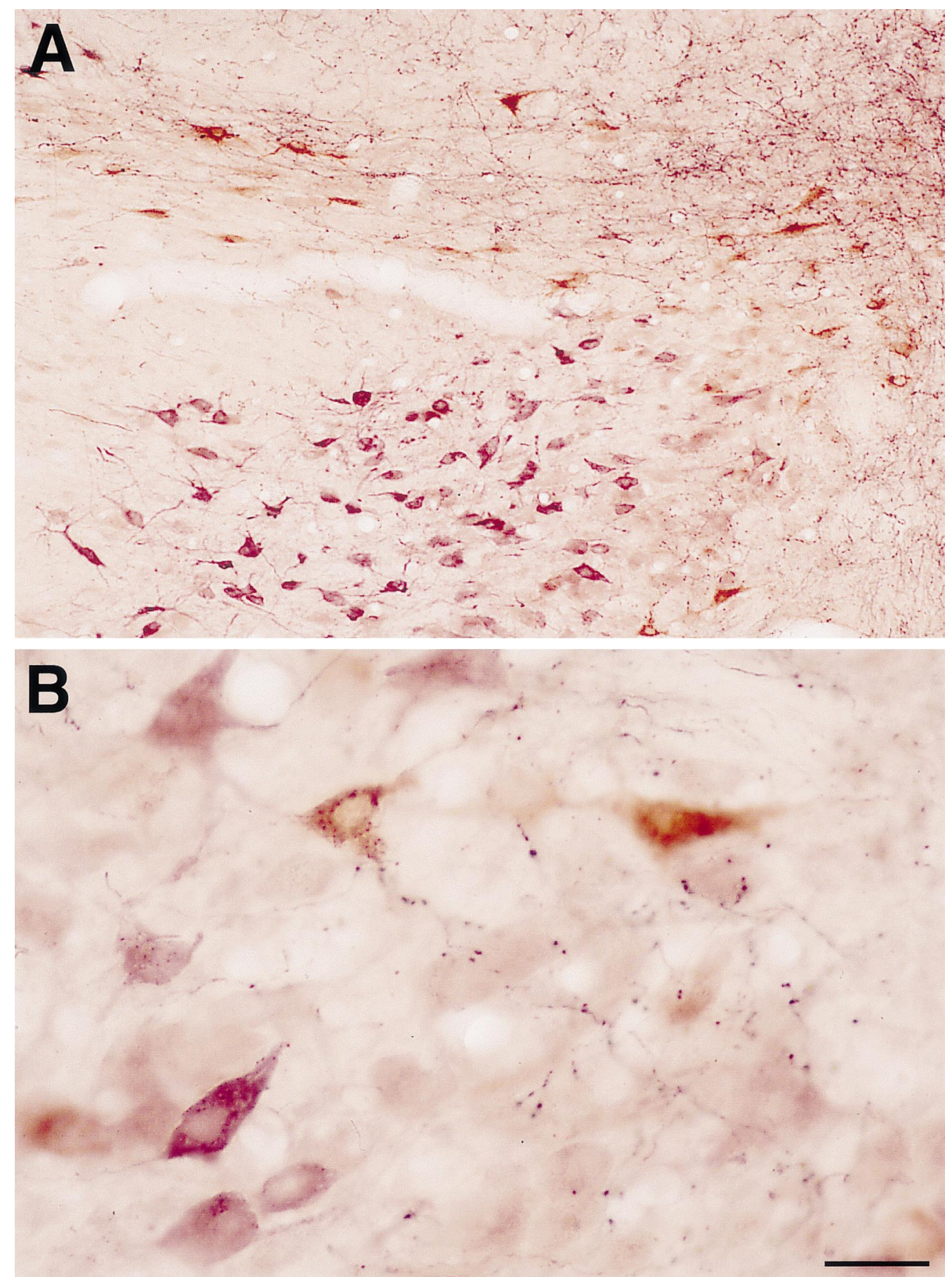

Fig. 2. Triple neuroanatomical tracing in a primate, Macaca fascicularis, by combining single anterograde BDA tracing with dual retrograde tract-tracing with CTB and FG. (A) Photomicrograph taken from the macaque substantia nigra. Brown-stained cells are a subpopulation of CTB-labeled, nigrostriatal neurons projecting to the putamen. A different subpopulation of nigrostriatal neurons is labeled purple with FG, representing the neurons projecting to the caudate nucleus. BDA anterogradely-labeled fibers (coming from the globus pallidus) are stained black with $\mathrm{DAB}-\mathrm{Ni}$, and are distributed within the nigral territories containing CTB-labeled cells. (B) High-power photomicrograph taken from the substantia nigra of the same section, in order to better illustrate the final quality of the present procedure. BDA-labeled axonal varicosities can easily be noticed. Concerning the subcellular localization of the retrogradely-transported CTB and FG, please note that both tracers are largely confined to the cytoplasm, without being present in the cell nuclei. Scale bar is $300 \mu \mathrm{m}$ for Fig. 2A and $35 \mu \mathrm{m}$ for Fig. $2 \mathrm{~B}$.

the entorhinal cortex (neurons projecting to the hippocampus) and/or inhibitory entorhinal interneurons. We have chosen to visualize the perirhinal afferents to the entorhinal cortex via iontophoretical injections of BDA in the perirhinal cortex. Entorhinal principal cells in layer II were labeled by means of retrograde transport with FG (injected by iontophoresis in hippocampal field CA1 and stained in brown with 
DAB). Finally, a subpopulation of layer III entorhinal inhibitory interneurons was labeled using antibodies against parvalbumin. BDA labeled fibers of perirhinal origin were seen to contact almost exclusively inhibitory entorhinal interneurons containing the calcium binding protein, parvalbumin (Fig. 3B).
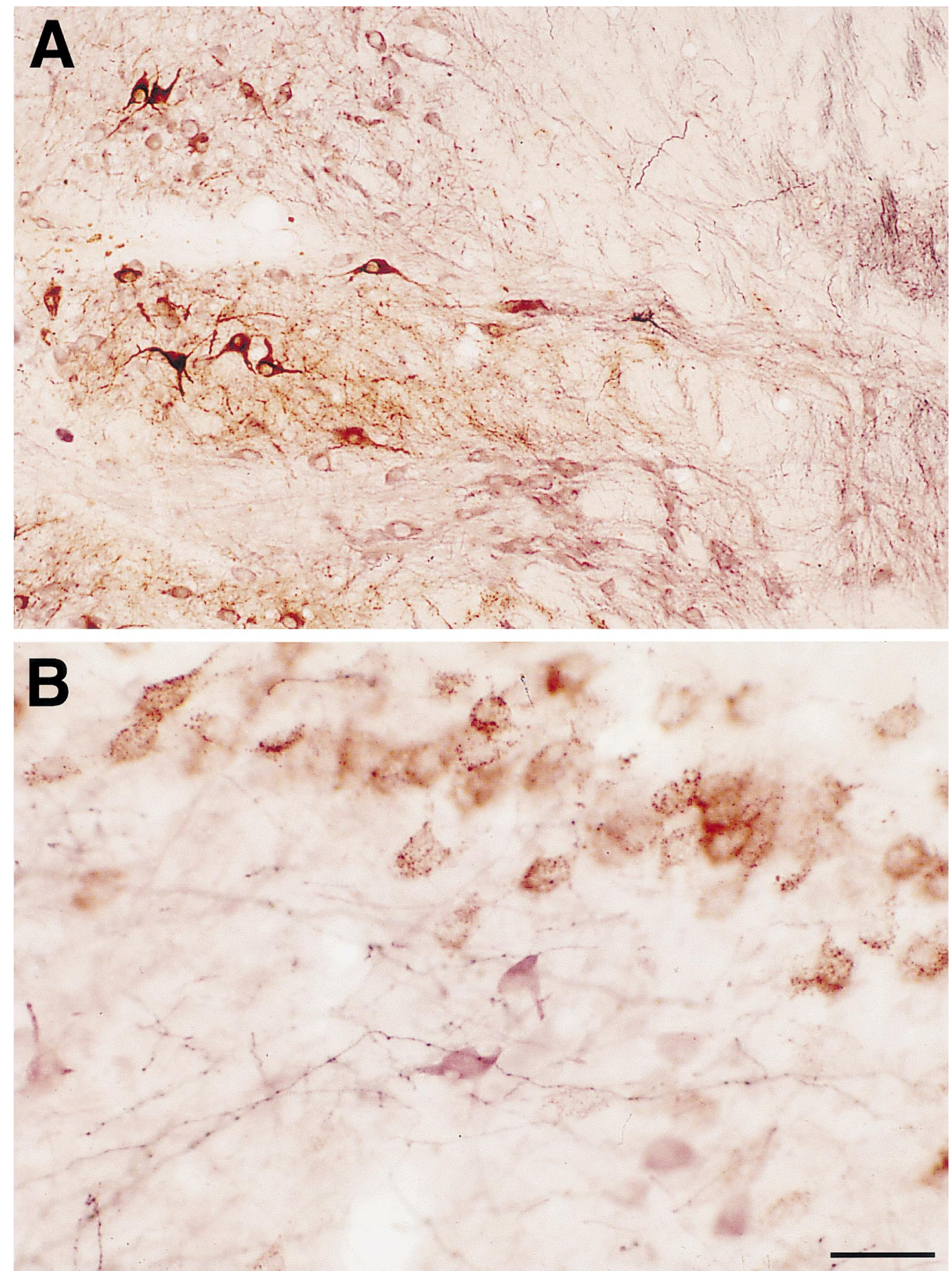

Fig. 3. Triple-staining procedures combining dual neuroanatomical tracing with the immunocytochemical detection of neuroactive substances. (A) Photomicrograph taken from the substantia nigra of a primate Macaca fascicularis illustrating the combination of anterograde tracing with BDA (black fibers), retrograde tracing with CTB (brown neurons) and the immunocytochemical detection of neurons containing tyrosine hydroxylase (purple). Since CTB is a bi-directionally transported marker, the section shows not only labeled neurons projecting to the caudate nucleus, but also afferent fibers (brown-labeled neuropil) coming from neurons located in the caudate nucleus (caudo-nigral projections). Nevertheless, the morphological quality of CTB-labeled axons is always less excellent than the quality obtained with BDA. (B) Photomicrograph taken from the rat entorhinal cortex, illustrating a combination of anterogradely transported BDA (black fibers with varicosities), retrogradely transported FG (brown-labeled, layer II principal cells) and neurons containing the calcium binding protein parvalbumin (purple stained layer III cells). Scale bar is $300 \mu \mathrm{m}$ for Fig. 3A and $25 \mu \mathrm{m}$ for Fig. 3B. 
When 'navigating' through triple staining procedures, many reefs must be avoided in order to produce the necessary high quality of all the staining components involved. Two major issues in obtaining this high quality are associated with the fact that the triple staining procedure is a kind of cascade that includes three individual procedures. These major issues are background staining and color mixing phenomena. If anything goes wrong with each of the individual stains, then the final result will inevitably be poor. Unaffordable degrees of background staining almost always hamper the contrast between the different color precipitates. Background staining is often observed when one antigen requires prolonged incubation in its corresponding chromogen solution in order to get the best staining. The amount of background staining obtained as a result of the first reaction has to be added to that obtained once the second reaction is over, and both may enhance the non-specific background staining associated with the third, final immunoreaction. Background staining phenomena may prevent the correct visualization of the finest labeled structures. In order to avoid this pitfall we recommend the use of high-titer antisera against the selected antigens as well as a vigilant monitoring under the microscope of the progress of each reaction. Another critical drawback is the tendency of a peroxidase substrate for taking the color corresponding to another chromogen previously used. This undesired phenomenon is called 'color mixing'. The phenomenon is induced under some conditions, such as (i) crossreactions between some of the different antisera used in the multiple staining protocol; (ii) the existence of areas containing high concentrations of reaction product, for example injection sites; (iii) prolonged incubation in the chromogen solutions and; (iv) co-localization of two or three antigens within the same structure. The strategy to avoid color mixing consist of a careful selection of the antisera, choosing those raised in different and non-crossreacting animal species and preferably those that most intensely label the target structures. Once again, microscopical monitoring at intervals of the progress of the chromogen reactions is mandatory. If co-localization of markers is expected, one obviously should select a combination of markers which is compatible with this expectation. When the antigens to be detected are located in separate subcellular localization, co-localization is usually not a major problem (Lanciego and Giménez-Amaya, 1999).

Finally, the order in which the chromogens are being applied during the staining procedure is another key issue. To our experience, best results are achieved by using first the DAB-Ni solution, next the DAB solution and finally the V-VIP chromogen. Different sequences have been tested during the development of this threecolor paradigm, always resulting in the appearance of unaffordable degrees of background staining, and in color mixing phenomena.

\section{Acknowledgements}

Technical assistance was supplied by A. Moreno and M.A. Erdozain. Supported by CICYT, Departamento de Salud del Gobierno de Navarra, Fundación 'Marcelino Botín' and Universidad de Navarra.

\section{References}

Akintunde A, Buxton DF. Quadruple labeling of brain-stem neurons: a multiple retrograde fluorescent tracer study of axonal collateralization. J Neurosci Methods 1992;45:15-22.

Angelucci A, Clascá F, Sur M. Anterograde axonal tracing with the subunit B of cholera toxin: a highly sensitive immunohistochemical protocol for revealing the fine axonal morphology in adult and neonatal brains. J Neurosci Methods 1996;65:101-12.

Balercia G, Cheng S, Bentivoglio M. Electron microscopic analysis of fluorescent neuronal labeling after photoconversion. J Neurosci Methods 1992;45:87-98.

Bentivoglio M, Su HS. Photoconversion of fluorescent retrograde tracers. Neurosci Lett 1990;113:127-33.

Bolam JP. Experimental Neuroanatomy. Oxford: Oxford University Press, 1992.

Brandt HM, Apkarian AV. Biotin-dextran: a sensitive anterograde tracer for neuroanatomic studies in rat and monkey. $\mathbf{J}$ Neurosci Methods 1992;45:87-98.

Bruce K, Grofova I. Notes on a light and electron microscopic double-labeling methods combining anterograde tracing with Phaseolus vulgaris leucoagglutinin and retrograde tracing with cholera toxin subunit B. J Neurosci Methods 1992;45:23-33.

Chang HT, Kuo H, Whittaker JA, Cooper NGF. Light and electron microscopic analysis of projection neurons retrogradely labeled with Fluoro-Gold: notes on the application of antibodies to Fluoro-Gold. J Neurosci Methods 1990;35:31-7.

Cowan WM, Cuénod M. The Use of Axonal Transport for Studies of Neuronal Connectivity. Amsterdam: Elsevier, 1975.

Divac I, Mogensen J. Long-term retrograde labelling of neurons. Brain Res 1990;524:339-41.

Dolleman-vanderWeel MJ, Wouterlood FG, Witter MP. Multiple anterograde tracing combining Phaseolus vulgaris leucoagglutining with rhodamine- and biotin-conjugated dextran amine. J Neurosci Methods 1994;51:9-21.

Ericson H, Blomqvist A. Tracing of neuronal connections with cholera toxin subunit B: light and electron microscopic immunohistochemistry using monoclonal antibodies. J Neurosci Methods 1988;24:225-35.

Glover JC, Petursdottir G, Jansen JKS. Fluorescent dextran-amines used as axonal tracers in the nervous system of the chicken embryo. J Neurosci Methods 1986;18:243-54.

Görcs T, Leranth C, MacLusky NJ. The use of gold substituted silver-intensified diaminobenzidine (DAB) for simultaneous electron microscopic labeling of tyrosine hydroxylase and glutamic acid decarboxylase immunoreactivity in the rat medial preoptic area. J Histochem Cytochem 1986;34:1439-47.

Hancock MB. DAB-Nickel substrate for the differential immunoperoxidase staining of nerve fibers and fiber terminals. J Histochem Cytochem 1982;32:311-4.

Heimer L, Robards MJ. Neuroanatomical Tract-Tracing Methods. NY: Plenum Press, 1981.

Heimer L, Záborsky L. Neuroanatomical Tract-Tracing Methods. Recent Progress. NY: Plenum Press, 1991.

Hendrickson AE. Electron microscopic autoradiography: identification of origin of synaptic terminals in normal nervous tissue. Science 1969;165:194-6. 
Hsu HS, Soban E. Color modification of diaminobenzidine (DAB) precipitation by metallic ions and its application for double immunohistochemistry. J Histochem Cytochem 1982;30:1079-82.

Kristensson K, Olsson Y, Sjöstrand J. Axonal uptake and retrograde transport of exogenous proteins in the hypoglossal nerve. Brain Res 1971;32:399-406.

Lakos S, Basbaum AI. Benzidine hydrochloride as a chromogen for single- and double-label and electron microscopic immunocytochemical studies. J Histochem Cytochem 1986;34:1047-56.

Lanciego, J.L., Wouterlood, F.G. (1994). Dual anterograde axonal tracing with Phaseolus vulgaris leucoagglutinin (PHA-L) and biotinylated dextran amine (BDA). Neurosci. Prot., 94-050-06.

Lanciego JL, Giménez-Amaya JM. Notes on the combined use of V-VIP and DAB peroxidase substrates for the detection of colocalizing antigens. Histochem Cell Biol 1999;111:305-11.

Lanciego JL, Goede PH, Witter MP, Wouterlood FG. Use of peroxidase substrate Vector $^{\circledR}$ VIP for multiple staining in light microscopy. J Neurosci Methods 1997;74:1-7.

Lanciego JL, Luquin MR, Guillén J, Giménez-Amaya JM. Multiple neuroanatomical tracing in primates. Brain Res Prot 1998a;2:323-32.

Lanciego JL, Wouterlood FG, Erro E, Giménez-Amaya JM. Multiple axonal tracing: simultaneous detection of three tracers in the same section. Histochem Cell Biol 1998b;110:509-15.

Levey AI, Bolam JP, Rye DB, Hallanger AE, Demuth RM, Mesulam MM, Wainer BH. A light and electron microscopic method for sequential double antigen localization using diaminobenzidine and benzidine hydrochloride. J Histochem Cytochem 1986;34:144957.

Liposits Z, Görcs T, Torök A, Domány S, Sétaló G. Simultaneous localization of two different tissue antigens based on the silver intensified PAP-DAB and the traditional PAP-DAB methods. Acta Morphol Acad Sci Hung 1983;31:356-69.

Luppi PH, Sakai K, Salvert D, Fort P, Jouvet M. Peptidergic hypothalamic afferents to the cat nucleus raphe pallidus as revealed by double immunostaining technique using unconjugated cholera toxin as a retrograde tracer. Brain Res 1987;402:339-45.

Luppi PH, Fort P, Jouvet M. Iontophoretic application of unconjugated cholera toxin B subunit (CTB) combined with immunohistochemistry of neurochemical substances: a method for transmitter identification of retrogradely labeled neurons. Brain Res 1990;534:209-24.

Mesulam MM. The blue reaction product in horseradish peroxidase neurohistochemistry. J Histochem Cytochem 1976;24:1273-80.

Mesulam MM. Tetramethylbenzidine for horseradish peroxidase neurochemistry: a non-carcinogenic blue reaction product with superior sensitivity for visualizing neural afferents and efferents. J Histochem Cytochem 1978;26:106-17.

Norgren RB, Lehman MN. A double-label pre-embedding immunoperoxidase technique for electron microscopy using diaminobenzidine and tetramethylbenzidine as markers. J Histochem Cytochem 1989;37:1283-9.

Novikova L, Novikov L, Kellerth J-O. Persistent neuronal labeling by retrograde fluorescent tracers: a comparison between Fast Blue, Fluoro-Gold and various dextran conjugates. J Neurosci Methods 1997;74:9-15.

Pieribone VA, Aston-Jones G. The iontophoretic application of Fluoro-Gold for the study of afferents to deep brain nuclei. Brain Res 1988;475:259-71.

Rajakumar N, Elisevich K, Flumerfelt BA. Biotinylated dextran: a versatile anterograde and retrograde neuronal tracer. Brain Res 1993;607:47-53.
Reiner, A., Veenman, C.L., Honig, M.G. (1993). Anterograde tracing using biotinylated dextran amine. Neurosci. Prot., 93-050-14.

Rosene DL, Roy NJ, Davis BJ. A cryoprotection method that facilitates cutting frozen sections of whole monkey brain for histological and histochemical processing without freezing artifact. J Histochem Cytochem 1986;34:1301-16.

Schmued, L.C. (1994). Anterograde and retrograde neuroanatomical tract-tracing with fluorescent compounds. Neurosci. Prot., 94-05002 .

Schmued LC, Fallon JH. Fluoro-Gold: a new fluorescent retrograde axonal tracer with numerous unique properties. Brain Res 1986;377:147-54.

Schmued LC, Heimer L. Iontophoretic injection of fluorogold and other fluorescent tracers. J Histochem Cytochem 1990;38:721-3.

Smith Y, Bolam JP. Combined approaches to experimental neuroanatomy: combined tracing and immunocytochemical techniques for the study of neuronal microcircuits. In: Bolam JP, editor. Experimental Neuroanatomy, A Practical Approach. Oxford: Oxford University Press, 1992:239-66.

Stoeckel K, Schwab ME, Thoenen H. Role of gangliosides in the uptake and retrograde axonal transport of cholera and tetanus toxin as compared to nerve growth factor and wheat germ agglutinin. Brain Res 1977;132:273-85.

Trojanowski JQ, Gonatas JO, Gonatas NK. Conjugates of horseradish peroxidase (HRP) with cholera toxin and wheat germ agglutinin are superior to free HRP as orthograde transported markers. Brain Res 1981;223:381-5.

Trojanowski JQ, Gonatas JO, Steiber A, Gonatas NK. Horseradish peroxidase (HRP) conjugates of cholera toxin and lectins are more sensitive retrograde transported markers than free HRP. Brain Res 1982;231:33-50.

Trojanowski JQ, Obrocka MA, Lee VMY. A comparison of eight different chromogen protocols for the demonstration of immunoreactive neurofilaments of glial elements in the rat cerebellum using the anti-peroxidase method and monoclonal antibodies. J Histochem Cytochem 1983;31:1217-23.

Van den Pol AN. Silver-intensified gold and peroxidase as dual ultrastructural immunolabels for pre- and postsynaptic neurotransmitters. Science 1985;228:332-5.

Van Bockstaele EJ, Wright AM, Cestari DM, Pickel VM. Immunolabeling of retrogradely transported fluorogold. Sensitivity and application of ultrastructural analysis of transmitter-specific mesolimbic circuitry. J Neurosci Methods 1994;55:65-78.

Veenman CL, Reiner A, Honig MG. Biotinylated dextran as an anterograde tracer for single- and double-label studies. J Neurosci Methods 1992;55:65-78.

Vetter DE, Saldaña E, Mugnaini E. Input from the inferior colliculus to medial olivocochlear neurons in the rat: a double label study with PHA-L and cholera toxin. Hear Res 1993;70:173-86.

Wan XCS, Trojanowski JQ, Gonatas JO. Cholera toxin and wheat germ agglutinin conjugates as neuroanatomical probes: their uptake and clearance, transganglionic and retrograde transport and sensitivity. Brain Res 1982;243:215-24.

Wessendorf MW. Fluoro-Gold: composition, and mechanism of uptake. Brain Res 1991;553:135-48.

Wouterlood FG, Jorritsma-Byham B. The Anterograde neuroanatomical tracer biotinylated dextra amine: comparison with the tracer PHA-L in preparations for electron microscopy. J Neurosci Methods 1994;48:75-87.

Zhou M, Grofova I. The use of peroxidase substrate Vector ${ }^{\circledR}$ VIP in electron microscopic single and double antigen localization. J Neurosci Methods 1995;62:149-58. 